\title{
MiR-193b-5p inhibits proliferation and enhances radio-sensitivity by downregulating the AKT/mTOR signaling pathway in tongue cancer
}

\author{
Lipeng Jiang ${ }^{1,2}$, Chunyan $\mathrm{He}^{3}$, Xin Zhang ${ }^{2}$, Yan $\mathrm{Chen}^{4}$, Guang $\mathrm{Li}^{1}$ \\ ${ }^{1}$ Department of Radiotherapy, The First Hospital of China Medical University, Shenyang 110001, China; ${ }^{2}$ Department of Oncology, The First \\ Affiliated Hospital of Jinzhou Medical University, Jinzhou 121001, China; ${ }^{3}$ Department of Prosthodontics, The Second Affiliated Hospital of Jinzhou \\ Medical University, Jinzhou 121000, China; ${ }^{4}$ Department of Radiotherapy, Affiliated Hospital of Chifeng University, Chifeng 024000, China \\ Contributions: (I) Conception and design: L Jiang, C He; (II) Administrative support: G Li, L Jiang; (III) Provision of study materials or patients: L \\ Jiang, G Li; (IV) Collection and assembly of data: L Jiang, C He, X Zhang, Y Chen; (V) Data analysis and interpretation: L Jiang, C He, X Zhang, Y \\ Chen; (VI) Manuscript writing: All authors; (VII) Final approval of manuscript: All authors. \\ Correspondence to: Guang Li. Department of Radiotherapy, The First Hospital of China Medical University, No. 155 Nanjing Street, Heping district, \\ Shenyang 110001, China. Email: fsyyjlp@163.com.
}

Background: MicroRNAs (miRNAs) have been found to have functions regulating cell proliferation, differentiation and apoptosis, thereby regulating the occurrence, development and prognosis of tumors. MiR-193b-3p is well-known for its tumorigenic effect, but there are few studies on miR-193b-5p, and its role in tongue cancer has not been reported.

Methods: In the present research, we investigated the specific role of miR-193b-5p in tongue cancer. MiR-193b-5p mimics were transfected into tongue cancer cell lines CAL27 and TCA-8113 to generate miR-193b-5p overexpression cells. CCK-8, clonogenic assay, wound healing assay, transwell and flow cytometry analysis were performed to detect cell proliferation, migration, invasion and apoptosis.

Results: Our data showed that the exogenous overexpression of miR-193b-5p blocked the proliferation, inhibited the phosphorylation of AKT and mTOR, and downregulated the levels of Cyclin D1 and P70 of CAL27 and TCA-8113 cells. We predicted that miR-193b-5p suppressed the proliferation of cancer cells by inhibiting the AKT/mTOR pathway. MiR-193b-5p mimics also induced the apoptosis of CAL27 and TCA- 8113 cells by inhibiting the expression of $\mathrm{Bcl} 2$ and promoting the levels of Active-Caspase 3 and Bax. Furthermore, a marked decline in the migration and invasiveness of tongue cancer cells transected with miR-193b-5p mimics was observed. According to the results of western blot, miR-193b-5p downregulated the levels of the epithelial-to-mesenchymal transition (EMT) markers, including N-cad, Vimentin, Snail and Slug, while upregulating E-cad expression level in CAL27 and TCA-8113 cells, suggesting that miR-193b-5p inhibited the migration and invasion by reversing the EMT process. In addition, miR-193b-5p mimics inhibited the formation of clonogenic colonies of CAL27 and TCA-8113 cells after irradiation.

Conclusions: Taken together, miR-193b-5p mimics block cell proliferation, migration and invasion and induce apoptosis by inhibiting the AKT/mTOR signaling pathway; they also reversed EMT progression and inhibited the radio-resistance of tongue cancer cells. Our results provide a potential target for the clinical treatment of human tongue cancer.

Keywords: miR-193b-5p; tongue cancer; radio-sensitivity; proliferation; apoptosis

Submitted Oct 23, 2019. Accepted for publication Feb 04, 2020.

doi: $10.21037 /$ tcr.2020.02.14

View this article at: http://dx.doi.org/10.21037/tcr.2020.02.14 


\section{Introduction}

Tongue cancer is a malignant tumor that originates from the anterior part of the tongue and is the most common malignant tumor in the oral cavity (1). Tongue cancer can metastasize through lymphatic and blood circulation pathways. Patients with low differentiation are prone to recurrence and poor prognosis after operation (2). Therefore, exploring the molecular mechanism of tongue cancer development and reducing the incidence of tongue cancer is a major issue that needs to be urgently solved.

MicroRNAs (miRNAs) are endogenous noncoding small RNAs of approximately $22 \mathrm{nt}$ in length and are highly conserved in evolution and have posttranscriptional gene regulatory functions (3). With the deepening of miRNA research, they have been found to involve in regulating cell proliferation, differentiation and apoptosis, thereby regulating the occurrence, development and prognosis of tumors $(4,5)$. Calin et al. first reported on the relationship between miRNAs and tumors in 2002 (6). Since then, it has been found that miRNAs are abnormally expressed in a variety of tumors and play roles in tumor proliferation, differentiation, metastasis, and therapeutic response (7).

In recent years, studies have found that miRNAs are involved in the development of tongue cancer (8-10). Ectopic expression of miR-639 effectively blocked the epithelial-to-mesenchymal transition (EMT) process by regulating FOXC1 and transforming growth factor beta (TGFB) (11). The downregulation of the miRNA miR-193b can promote the invasion and metastasis of human breast cancer (12); ectopic expression of miR-193b can induce cell cycle arrest of human primary hepatocytes and inhibit the migration and invasion of tumor cells (13). The effect of miR-193b in various tumors has been gradually elucidated as a negative regulatory factor that can inhibit the proliferation, migration and invasion of cancer cells. It has been reported that miR-193b-3p expression indicates a good prognosis in head and neck squamous cell carcinomas (12). However, at present, there have been few studies of miR$193 \mathrm{~b}-5 \mathrm{p}$ in tumors, and its role and specific mechanism in tongue cancer progression have not been reported. We, therefore, investigated the specific effect of miR-193b-5p in tongue cancer through transfecting its mimics into the cell lines CAL27 and TCA-8113 to provide a potential target for the clinical treatment of human tongue cancer.

\section{Methods}

\section{Cell lines}

The human tongue cancer cell lines CAL27 and TCA8113 were cultured in DMEM with $10 \%$ FBS, $100 \mathrm{U} / \mathrm{mL}$ penicillin and $0.1 \mathrm{mg} / \mathrm{mL}$ streptomycin at $37{ }^{\circ} \mathrm{C}$ with $5 \%$ $\mathrm{CO}_{2}$. The cells were trypsinized at the logarithmic growth phase and seeded into six-well plates for subsequent experiments. When the cell density reached approximately $80 \%$, the cells were transfected with miR-193b-5p mimics (CGGGGUUUUGAGGGCGAGAUGA, RIBOBIO, China) or negative control miRNA using Lipofectamine 2000 (Invitrogen, USA).

\section{Cell proliferation assay}

Cell counting kit-8 (CCK-8, Solarbio Science and Technology Ltd., China) was used for the determination of cell proliferation. Twenty-four hours after transfection, the cells were seeded in a 96-well plate at 1,000 cells per well. The cell viability was measured every $24 \mathrm{~h}$. Before detection, $10 \mu \mathrm{L}$ CCK-8 reagent was added to each well and incubated at $37^{\circ} \mathrm{C}$ for $1.5 \mathrm{~h}$. OD value was measured at $450 \mathrm{~nm}$ by an enzyme labeling instrument, and the proliferation curve was plotted. Each experiment was performed in triplicate.

\section{Clonogenic assay}

After $24 \mathrm{~h}$ of transfection, cells were seeded into $60 \mathrm{~mm}$ dishes at a concentration of 500 cells per plate. The cells were cultured at $37{ }^{\circ} \mathrm{C}$ and $5 \% \mathrm{CO}_{2}$ for $1-2$ weeks. When clones are visible to the naked eye in dishes, the culture was terminated. Cells were fixed with $5 \mathrm{~mL} 4 \%$ paraformaldehyde for $30 \mathrm{~min}$. Then, $0.1 \%$ crystal violet dye solution was added and incubated for $30 \mathrm{~min}$. The number of clones larger than 50 cells was counted under the microscope.

\section{Wound bealing assay}

The migration of CAL27 and TCA-8113 was detected by wound healing assay. After $24 \mathrm{~h}$ of transfection, approximately $5 \times 10^{5}$ cells were added to the six-well plate and cultured overnight. Scratches were made on the cells 
with a pipette tip. The cells were washed with PBS three times. Serum-free medium was added, and cells were cultured at $37{ }^{\circ} \mathrm{C}$ and $5 \% \mathrm{CO}_{2}$. Photographs were taken at 0 and $24 \mathrm{~h}$ after scratch. ImageJ software was used to analyze the percentage of wound closure.

\section{Transwell assay}

Matrigel was diluted with precooled serum-free medium. We added $100 \mu \mathrm{L}$ Matrigel to the transwell chamber, which was incubated at $37^{\circ} \mathrm{C}$ for $4 \mathrm{~h}$. Twenty-four hours after transfection, cells were cultured in serum-free medium to prepare a cell suspension. $100 \mu \mathrm{L}$ of cell suspension (approximately $1 \times 10^{5}$ cells) was added to the upper chamber and $500 \mu \mathrm{L}$ complete medium was added to the lower chamber. After $24 \mathrm{~h}$ of incubation, cells were fixed with $5 \mathrm{~mL} 4 \%$ paraformaldehyde for $30 \mathrm{~min}$. Then, $0.1 \%$ crystal violet dye solution was added and incubated for $30 \mathrm{~min}$. Photomicrographs were captured, and the number of cells was counted.

\section{Fluorescence quantitative PCR (qPCR)}

Total RNA of each group was extracted using Ultrapure RNA Kit (CW0581, CWBIOTECH, China), and cDNA was synthesized with miRNA cDNA Synthesis Kit (CW2141S, CWBIOTECH, China). The expression of miR-193b was detected by using a miRNA qPCR Assay Kit (CW2142S, CWBIOTECH, China). The primers were listed as below: miR-193b: 5'- CGGGGTTTTGAGGGCGAGA -3'. The expression volume was calculated by the $2^{-\Delta C t}$ method from 3 independent experiments.

\section{Western blotting}

After $48 \mathrm{~h}$ of cell transfection, the total protein was extracted with RIPA lysate (plus protease inhibitor). The protein concentration was determined by the BCA method. Twenty micrograms of protein were added into each well, SDS polyacrylamide gel electrophoresis was used to isolate protein samples, and then the protein was transferred to a PVDF membrane. Then, the membrane was blocked with $5 \%$ skimmed milk and incubated overnight at $4{ }^{\circ} \mathrm{C}$ with primary antibodies, followed by secondary antibodies at room temperature for $1 \mathrm{~h}$. Primary antibodies in this study were purchased from Proteintech, including Bcl2 (127891-AP, 1:1,000), Caspase3 (19677-1-AP, 1:1,000), Cleaved-
Caspase3 (19677-1-AP, 1:1,000), Bax (50599-2-Ig, 1:1,000), AKT (10176-2-AP, 1:1,000), p-AKT (66444-1-Ig, 1:1,000), Cyclin D1 (60186-1-Ig, 1:1,000), P70(14485-1-AP, 1:1,000) and GAPDH (10494-1-AP, 1:4,000). Primary antibodies for mTOR (\#2972, 1:1,000) and p-mTOR (\#5536, 1:1,000) were purchased from cell signaling. ECL substrate reagent was used to generate chemiluminescent signals. QUANTITY ONE software was used to scan gray values, GAPDH was used as an internal reference, and the relative expression of protein was calculated by target protein/ internal reference.

\section{Flow cytometry analysis}

After transfection, cells were cultured in serum-free medium for $24 \mathrm{~h}$. Cell density was adjusted to $1-5 \times 10^{6}$ cells $/ \mathrm{ml}$ by binding buffer. One hundred $\mu \mathrm{L}$ of cell suspension was incubated with $5 \mu \mathrm{L}$ Annexin V/FITC (FXP018-100, 4A Biotech Company, China) at room temperature for $5 \mathrm{~min}$, then $10 \mu \mathrm{L}$ PI staining was added, and cell apoptosis was detected. Flowjo software was used to analyze the results.

\section{Statistical analyses}

Statistical analysis was performed using SPSS 18.0 software. The comparison between the miR-193b-5p and NC groups was performed by using $t$-test. Data were displayed as the mean \pm SD. Differences were considered statistically significant for values of $\mathrm{P}<0.05$.

\section{Results}

Overexpression of miR-193b-5p blocked the proliferation by the inbibition of the AKT/mTOR signaling patbway in tongue cancer cells

To investigate the specific role of miR-193b-5p in human tongue cancer, we overexpressed it in two tongue cancer cell lines, CAL27 and TCA-8113, by the transfection of miR-193b-5p mimics, with the negative control miRNA as a control group. After incubation for $24 \mathrm{~h}$, the total RNA was extracted and miR-193b-5p expression level was determined by qPCR. As shown in Figure $1 A, B$, miR-193b-5p mimics markedly increased the expression of miR-193b-5p compared with the control cells $(\mathrm{P}<0.05)$. The effect of miR-193b-5p on cell proliferation was detected by CCK-8 and clonogenic assays. As shown in Figure 1C,D, transfection of miR-193b-5p mimics significantly decreased 
A
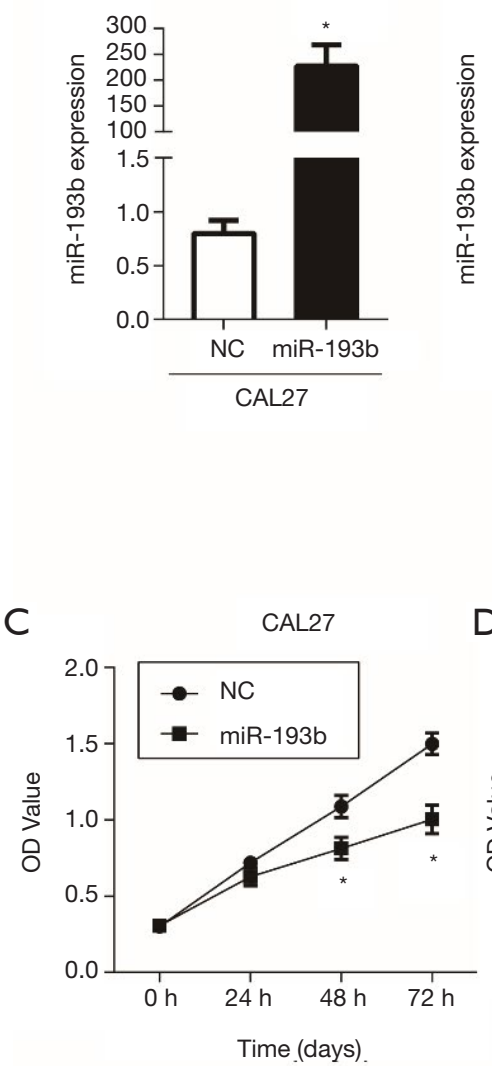

B

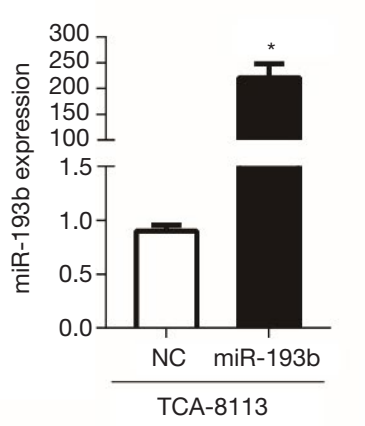

TCA-8113

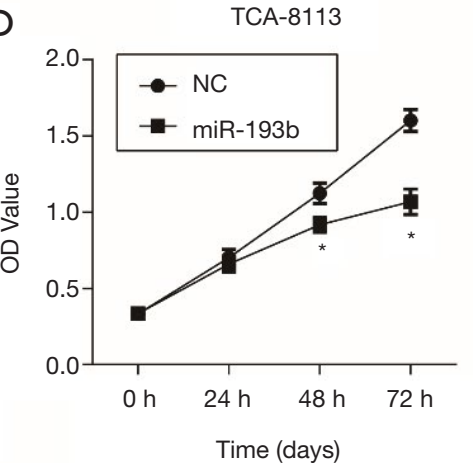

$E$

CAL27
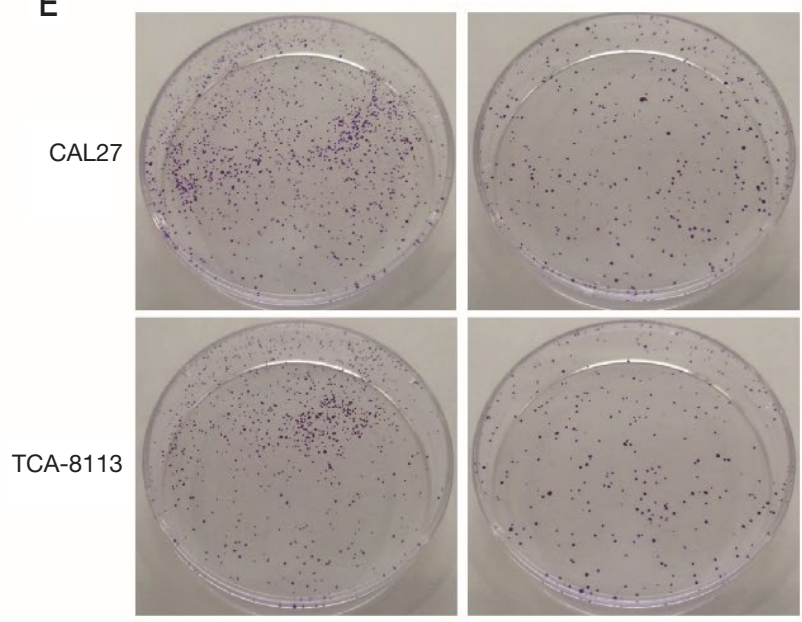

NC

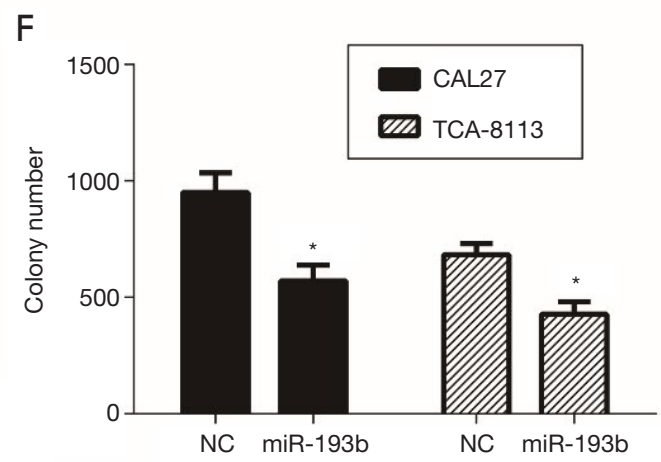

Figure 1 MiR-193b-5p inhibited the proliferation of tongue cancer cells. CAL27 (A) and TCA-8113 (B) cells were transfected with miR193b-5p mimics to generate miR-193b-5p overexpression cells (miR-193b-5p), and the expression of miR-193b-5p was determined using qPCR. The cells transfected with negative control miRNA was as a control group (NC). Cell proliferation was detected using CCK-8 assay. Transfection of miR-193b-5p mimics significantly decreased the OD value of CAL27 (C) and TCA-8113 (D); (E,F) the proliferation was further confirmed using colony formation assay. Cells were stained with $0.1 \%$ crystal violet dye. Colony number of miR-193b-5p overexpressed cells markedly decreased compared with that of control cells. All experiments were repeated three times. * $\mathrm{P}<0.05$.

the OD value of CAL27 and TCA-8113. The results of clonogenic assay showed that the colony number of miR-193b-5p-overexpressing cells was markedly decreased compared with that of control cells (Figure $1 E, F, \mathrm{P}<0.05)$. All the results suggested that miR-193b-5p blocked the proliferation in human tongue cancer cells.

Previous studies have shown that miR-193b-5p can inhibit tumor progression by regulating AKT activity in a variety of tumors $(14,15)$. Therefore, we detected the expressions of the AKT/mTOR signaling pathway-related genes AKT, p-AKT, mTOR, p-mTOR, Cyclin D1 and P70 using western blot. From our results, miR-193b-5p inhibited the phosphorylation of AKT and mTOR; it also downregulated the levels of Cyclin D1 and P70, two downstream proteins of $\mathrm{AKT} / \mathrm{mTOR}$ signaling pathway, in both CAL27 and TCA-8113 cells (Figure 2, $\mathrm{P}<0.05$ ). The AKT/mTOR pathway is closely associated with lots of processes of cells, including angiogenesis, cell survival and growth. These data suggested that miR-193b-5p blocked proliferation by activating the AKT/mTOR signaling pathway in human tongue cancer cells.

\section{Overexpression of miR-193b-5p inbibited the migration and invasion of tongue cancer cells}

Next, we investigated the effect of miR-193b-5p on migration and invasion in CAL27 and TCA- 8113 cells using wound healing and transwell assays, respectively. The 
A

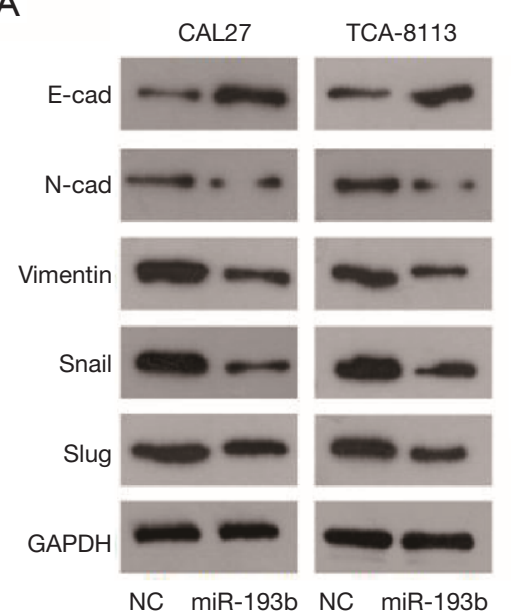

D

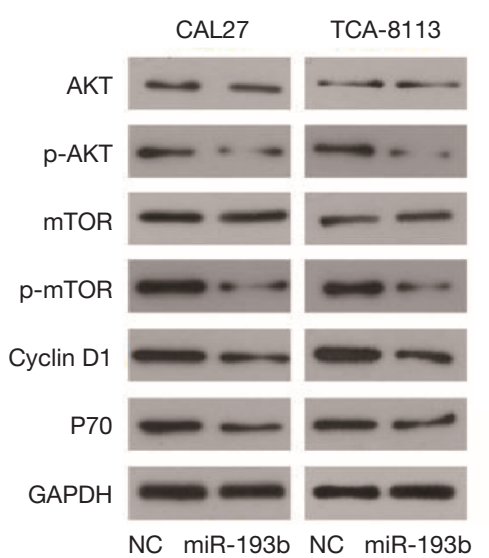

B

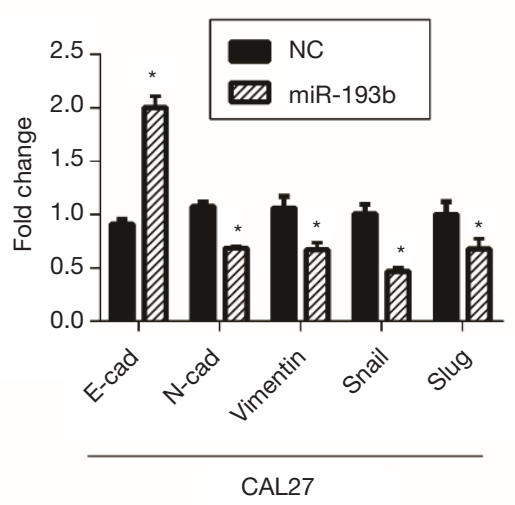

E

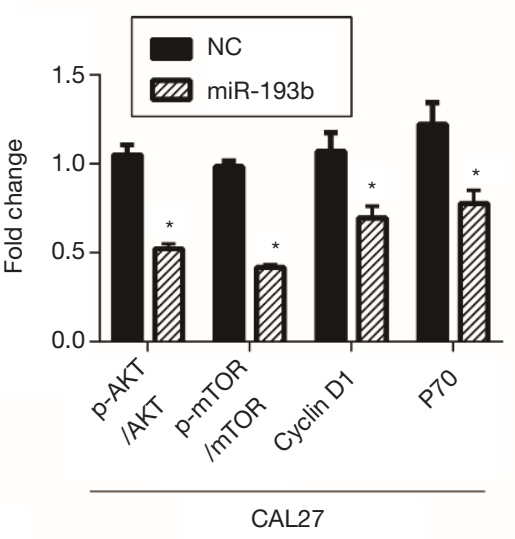

C

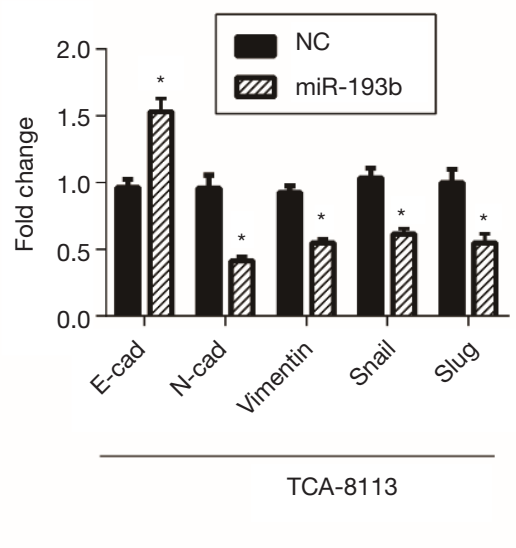

$\mathrm{F}$

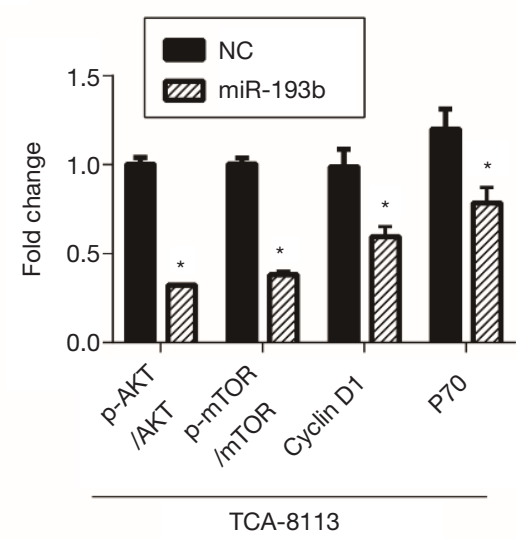

Figure 2 MiR-193b-5p inhibited EMT progress and the activation of the AKT/mTOR signaling pathway in tongue cancer cells. (A) The expression levels of the EMT-related genes E-cad, N-cad, Vimentin, Snail and Slug were detected by western blot and normalized to GAPDH density; miR-193b-5p downregulated the levels of N-cad, Vimentin, Snail and Slug while upregulating E-cad expression levels in CAL27 (B) and TCA-8113 (C) cells; (D) the expression levels of the AKT/mTOR signaling pathway-related genes AKT, p-AKT, mTOR, p-mTOR, Cyclin D1 and P70 were detected by western blot and normalized to GAPDH density; miR-193b-5p downregulated the levels of p-AKT/AKT, p-mTOR/mTOR, Cyclin D1 and P70 in CAL27 (E) and TCA-8113 (F) cells. Three repetitions for each group were performed. *, $\mathrm{P}<0.05$.

representative micrographs of wound healing assay were shown in Figure $3 A$. In comparison to the control group, the percentage of wound closure was significantly reduced in cells with the transfection of miR-193b-5p mimics (Figure $3 B, \mathrm{P}<0.05$ ). Then, transwell assay was performed to investigate the role of miR-193b-5p on cell invasion. As shown in Figure 3C,D, overexpression of miR-193b-5p reduced the invasion cell number in both CAL27 (196 \pm 57$)$ and TCA-8113 (119 \pm 24$)$ cells compared with the respective control group (CAL27, 354 \pm 68 ; TCA-8113, 231 \pm 56 )
$(\mathrm{P}<0.05)$. These data demonstrated that miR-193b-5p inhibited migration and invasion in human tongue cancer cells.

\section{Overexpression of miR-193b-5p promoted apoptosis in tongue cancer cells}

The occurrence and development of tumors is not only the result of uncontrolled cell proliferation and abnormal cell differentiation but is also related to the imbalance of tumor cell apoptosis. Apoptosis plays a crucial role in 
A

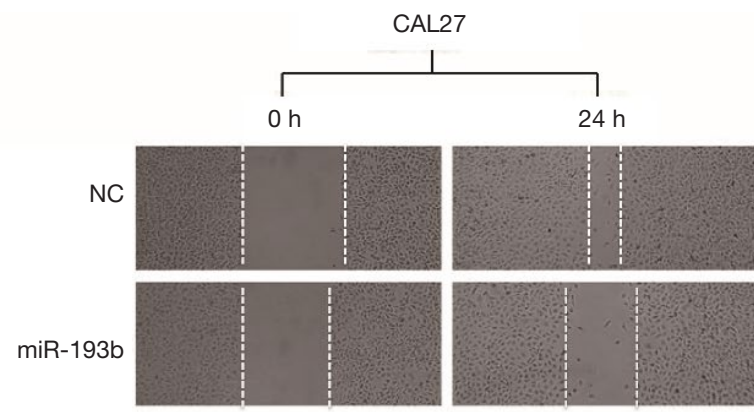

B

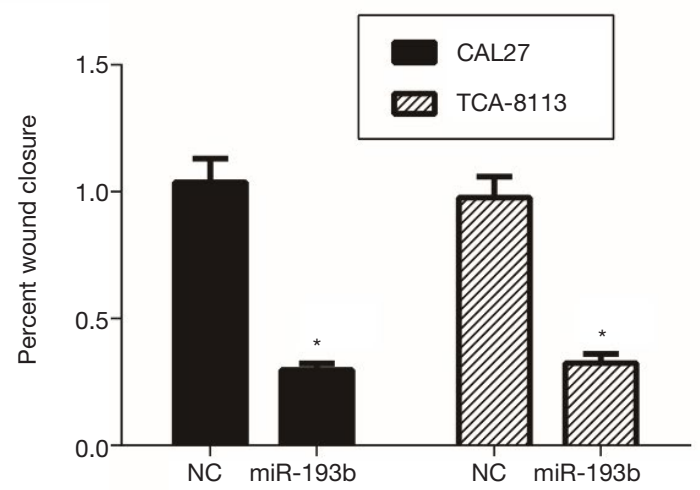

C

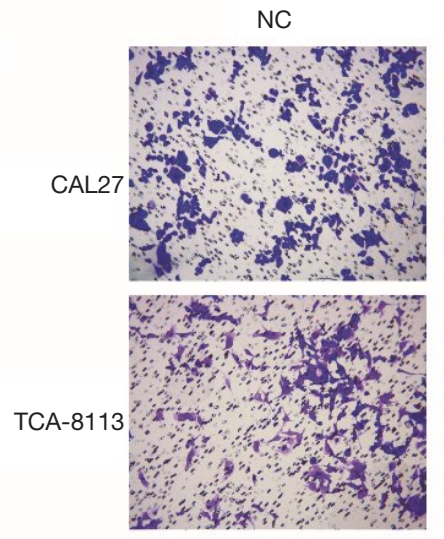

TCA-8113
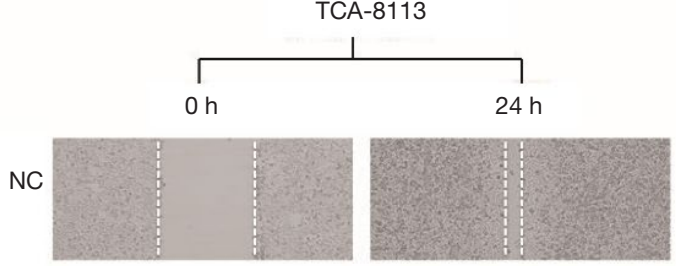

miR-193b
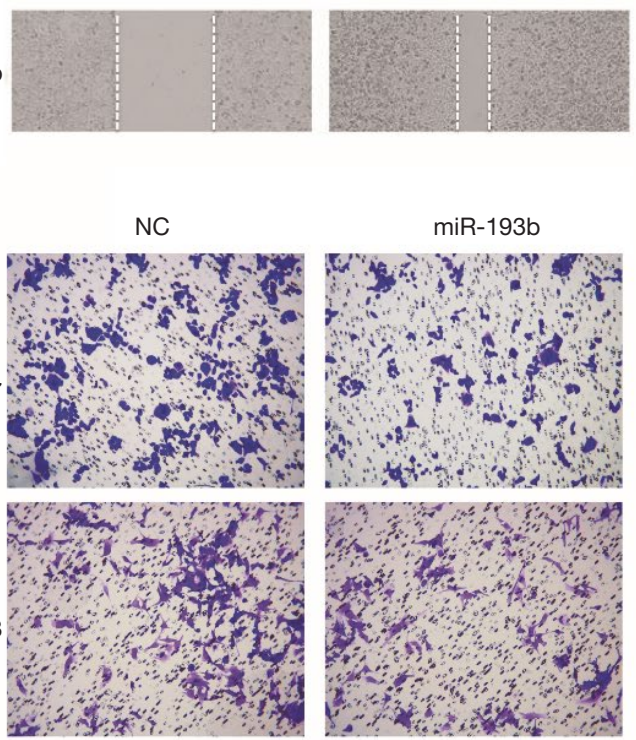

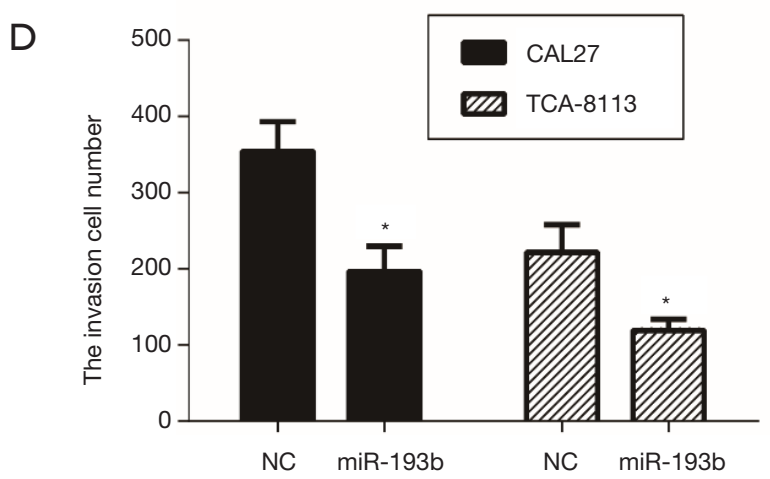

Figure 3 MiR-193b-5p inhibited migration and invasion in tongue cancer cells. (A) The migration of CAL27 and TCA-8113 cells was detected using wound healing assay (magnification: 100x); (B) in comparison to the control group, the percentage of wound closure was significantly reduced in cells with the transfection of miR-193b-5p mimics; (C) transwell assay was performed to investigate the role of miR193b-5p on cell invasion; Cells were stained with $0.1 \%$ crystal violet dye (magnification: $200 \times$ ). (D) the invasion cell number was counted and shown in the column diagram. Overexpression of miR-193b-5p reduced the invasion cell number compared with the control group. All experiments were repeated three times. *, $\mathrm{P}<0.05$.

the regulation of tumorigenesis and can inhibit the rapid growth of tumor cells (16). Therefore, we investigated the role of miR-193b-5p mimics on apoptosis by flow cytometry assay in CAL27 and TCA-8113 cells. As shown in Figure $4 A, B, C$, the percentage of apoptotic cells increased after the overexpression of miR-193b-5p in both CAL27 and TCA-8113 cells. Then, the expressions of the apoptosisrelated genes Bcl2, Caspase3, Cleaved-Caspase3 and Bax were detected by western blot and normalized to GAPDH. As shown in Figure 4D,E,F, miR-193b-5p mimics inhibited the expression of $\mathrm{Bcl} 2$ and promoted the levels of CleavedCaspase 3 and Bax in CAL27 and TCA-8113 cells $(\mathrm{P}<0.05)$. 
A

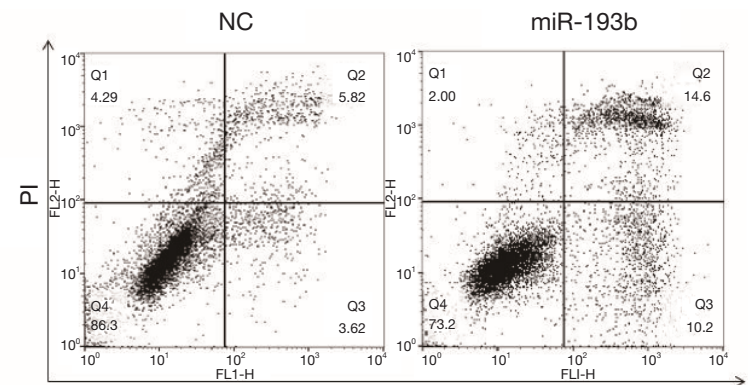

AnnexinV FITC

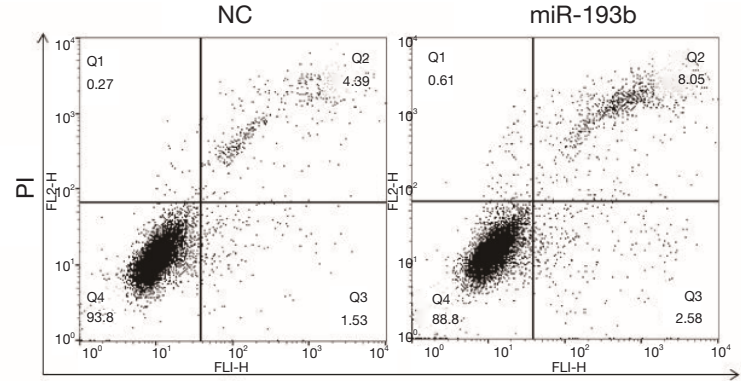

AnnexinV FITC

$\mathrm{D}$



E



C



$\mathrm{F}$



Figure 4 MiR-193b-5p promoted apoptosis in tongue cancer cells. (A) Apoptosis was determined by flow cytometry assay, and FlowJo software was used for data analysis. The percentage of apoptotic cells increased after the overexpression of miR-193b-5p in both CAL27 (B) and TCA-8113 (C) cells; (D,E,F) the expression levels of the apoptosis-related genes Bcl2, Active-Caspase3 and Bax were detected by western blot and normalized to GAPDH density. MiR-193b-5p inhibited the expression of Bcl2 and promoted the levels of Active-Caspase3 and Bax in CAL27 and TCA-8113 cells. All experiments were repeated three times. *, $\mathrm{P}<0.05$.

\section{MiR-193b-5p reversed EMT progress in tongue cancer cells}

EMT plays an important role in embryonic development and exists in the process of invasion and metastasis of tumors. During the process of tumorigenesis, normal epithelial cells undergo EMT and then develop into carcinoma in situ. Tumor cells can be locally diffused through EMT, and cells invade lymphatics and vessels to metastasize (17). In the present study, we detected the expressions of the EMT-related genes E-cad, N-cad, Vimentin, Snail and Slug in miR-193b-5poverexpressing cells by western blot. As shown in Figure 2A,B,C, miR-193b-5p downregulated the levels of N-cad, Vimentin, Snail and Slug while upregulating E-cad expression levels in both CAL27 and TCA-8113 cells. These results indicated that miR-193b-5p reversed EMT progress in human tongue cancer cells. 
A

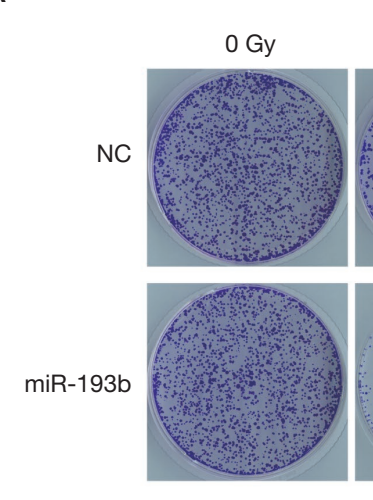

CAL27

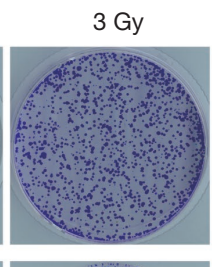



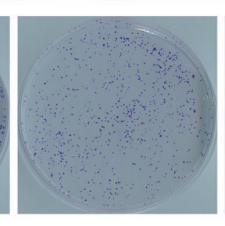

TCA-8113

C

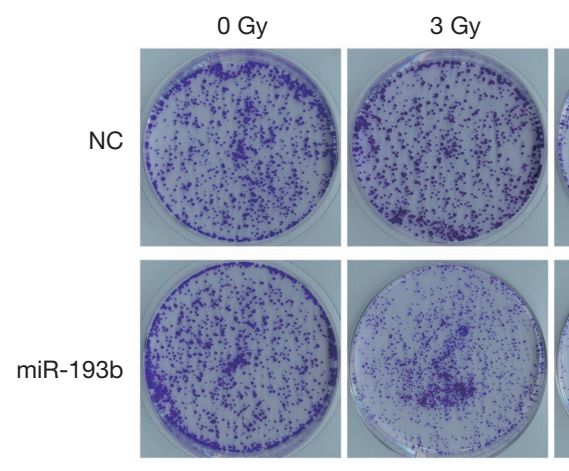

6 Gy

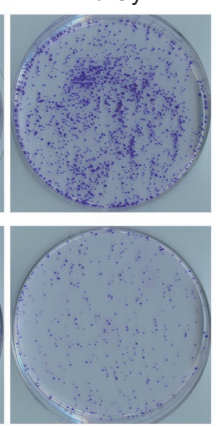

9 Gy

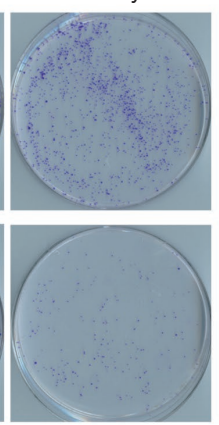

B



D

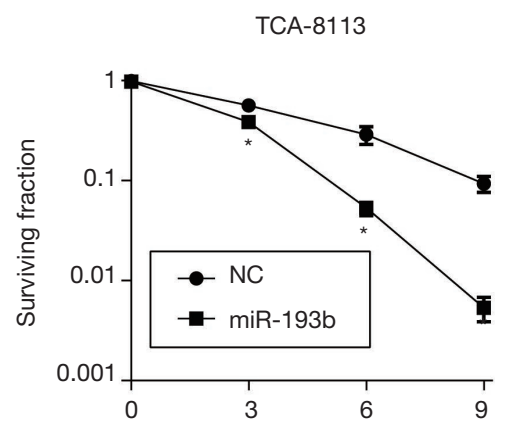

Figure 5 miR-193b-5p inhibited radio-resistance in tongue cancer cells. The radio-resistance of CAL27 (A,B) and TCA-8113 (C,D) cells with transfection were determined by using clonogenic assay after irradiation. Surviving fractions of cells were calculated 2 weeks later. MiR193b-5p mimics inhibited the formation of clonogenic colonies in both CAL27 and TCA-8113 cells after irradiation. Cells were stained with $0.1 \%$ crystal violet dye. All experiments were repeated three times. * $\mathrm{P}<0.05$.

\section{Overexpression of miR-193b-5p enhanced radio-sensitivity in tongue cancer cells}

Radiotherapy is one of the most important treatments for tongue cancer, but its effectiveness is limited by the radioresistance of tumor cells. Therefore, increasing the radiosensitivity of tongue cancer cells is a clinical focus to be solved urgently. Studies have reported that exogenous gene transfection can effectively increase the sensitivity of tumor cells to radiotherapy (18). In this research, we investigated the effect of miR-193b-5p on the radio-resistance of tongue cancer cells by using clonogenic assay after irradiation. As shown in Figure 5, surviving fractions of cells were calculated 2 weeks after the transfection. MiR-193b-5p mimics inhibited the formation of clonogenic colonies in both CAL27 and TCA-8113 cells after irradiation $(\mathrm{P}<0.05)$, suggesting that miR-193b-5p enhanced radio-resistance in human tongue cancer cells.

\section{Discussion}

The miR-193b-5p-encoding gene is located in human 16p13.12 and encodes a small noncoding molecule RNA. The specific binding of miR-193b-5p to the 3'UTR of the target gene inhibits protein translation; thus, miR-193b-5p participates in various pathological and physiological processes of the body and is an important regulatory factor $(12,19,20)$.

In the present research, we demonstrated for the first time that miR-193b-5p could inhibit the progression of tongue cancer. MiR-193b-5p mimics inhibited proliferation via promoting apoptosis in the tongue cancer cell lines CAL27 and TCA-8113. Previous studies have shown that miR-193b$5 \mathrm{p}$ acts as a tumor suppressor in a variety of tumors. Rauhala 
et al. found that miR-193b-5p was methylated in the prostate cancer (PC) cell line 22Rv1 at a CpG island $\sim 1 \mathrm{~kb}$ upstream of the miRNA locus, and its overexpression caused a significant growth inhibition resulting from a decrease of S-phase cells in the cell cycle (21). MiR-193b-5p is downregulated in pancreatic cancer tissues compared to adjacent healthy tissues and induced significantly decreased proliferative, migratory, and invasive capabilities in pancreatic cancer cells (22).

$\backslash$ MiR-193b-5p also induces non-apoptotic cell death and autophagy in esophageal cancer (23). Moreover, miR-193b$5 \mathrm{p}$ directly targets Cyclin D1 according to the reporter assay, thus inhibiting the proliferation of PC cells (24). It has been reported that microRNA-193b can inhibit cell proliferation in many other tumors, such as lung cancer, gastric cancer and liver cancer. Our results further confirm the anti-cancer effect of miR-193b-5p in solid tumors.

Our results demonstrated that miR-193b-5p overexpression promoted migration and invasion and reversed EMT progress in tongue cancer cells. Cell migration is a ubiquitous life phenomenon in organisms and plays a key role in tumor cell invasion and metastasis. At the same time, cells that have undergone EMT secrete large amounts related enzymes, which can lead to cell proliferation, morphological changes and the acquisition of metastatic ability (25). Studies have shown that miR-193b-5p inhibits cell infiltration and metastasis in a variety of tumors. Bioinformatics studies showed that there was a target site of miR-193b-5p in the urokinase-type plasminogen activator (uPA) 3'UTR (26). Anti-miR-193b$5 \mathrm{p}$ treatment resulted in the enhancement of uPA protein and increased the invasiveness of the human breast cancer cell line MDA-MB-231 (26). In mouse models, miR-193b-5p significantly inhibited the growth and metastasis of tumors. However, the role of miR-193b-5p in the EMT process is rarely reported. Based on the results of this study, we speculate that miR-193b-5p may inhibit cell migration and infiltration by reversing EMT.

Finally, we found that miR-193b-5p enhanced the radioresistance according to the results of clonogenic assay after irradiation. Han et al. reported that the competitive silencing of miR-193b-5p by lncRNA NEAT1 could facilitate radioresistance in cervical cancer cells (27). In addition, miR-193b$5 \mathrm{p}$ has been proven to enhance the response to anticancer drugs in tumors. The inhibition of miR-193b-5p expression makes $\mathrm{HBV}$-related HCC cells sensitive to sorafenib treatment and promotes the apoptosis induced by sorafenib (28). In human breast cancer, miR-193b-5p directly targeted the MCL-1 3'UTR and sensitized MCF-7/DOXR cells to doxorubicin (29). In esophageal cancer cells, miR-193b-5p mimics markedly blocked the recovery of KYSE450 cells following 5-fluorouracil
(5-FU) treatment (23). Our results further confirm the role of miR-193b-5p in assisting in the clinical treatment of tumors.

In conclusion, miR-193b-5p mimics block cell proliferation, migration and invasion and induce apoptosis by inhibiting the AKT/mTOR signaling pathway in human tongue cancer cells. MiR-193b-5p also reverses the EMT progress and inhibits the radio-resistance of tongue cancer cells. Our results contribute to expanding the role of miR-193b-5p in promoting tumor progression and provide a potential target for the clinical treatment of human tongue cancer.

\section{Acknowledgments}

Funding: This work was supported by the Natural Science Foundation Project of Liaoning Science and Technology Department (Nos 20170540340).

\section{Footnote}

Conflicts of Interest: All authors have completed the ICMJE uniform disclosure form (available at http://dx.doi. org/10.21037/tcr.2020.02.14). The authors have no conflicts of interest to declare.

Ethical Statement: The authors are accountable for all aspects of the work in ensuring that questions related to the accuracy or integrity of any part of the work are appropriately investigated and resolved. The study was conducted in accordance with the Declaration of Helsinki (as revised in 2013). The study was approved by the Ethics Committee of The First Affiliated Hospital of Jinzhou Medical University (IRB number: 202008). Informed consent was waived.

Open Access Statement: This is an Open Access article distributed in accordance with the Creative Commons Attribution-NonCommercial-NoDerivs 4.0 International License (CC BY-NC-ND 4.0), which permits the noncommercial replication and distribution of the article with the strict proviso that no changes or edits are made and the original work is properly cited (including links to both the formal publication through the relevant DOI and the license). See: https://creativecommons.org/licenses/by-nc-nd/4.0/.

\section{References}

1. Huang Z, Xie J, Lin S, et al. The downregulation of ANGPTL4 inhibits the migration and proliferation of tongue squamous cell carcinoma. Archives of Oral Biology 2016;71:144-9. 
2. Sessions DG, Spector GJ, Jason L, et al. Analysis of treatment results for oral tongue cancer. Laryngoscope 2002;112:616-25.

3. Jin J, Martin M, Hartley AV, et al. PRMTs and miRNAs: functional cooperation in cancer and beyond. Cell Cycle 2019;18:1676-86.

4. Liz J, Esteller M. IncRNAs and microRNAs with a role in cancer development. Biochim Biophys Acta 2016;1859:169-76.

5. Oliveto S, Mancino M, Manfrini N, et al. Role of microRNAs in translation regulation and cancer. World J Biol Chem 2017;8:45-56.

6. Calin GA, Dumitru CD, Shimizu M, et al. Frequent deletions and down-regulation of micro- RNA genes miR15 and miR16 at 13q14 in chronic lymphocytic leukemia. Proc Natl Acad Sci U S A 2002;99:15524-9.

7. Rupaimoole R, Calin GA, Lopez-Berestein G, et al. miRNA Deregulation in Cancer Cells and the Tumor Microenvironment. Cancer Discov 2016;6:235-46.

8. He Q, Chen Z, Cabay RJ, et al. microRNA-21 and microRNA-375 from oral cytology as biomarkers for oral tongue cancer detection. Oral Oncology 2016;57:15-20.

9. Rabinowits G, Bowden M, Flores LM, et al. Comparative Analysis of MicroRNA Expression among Benign and Malignant Tongue Tissue and Plasma of Patients with Tongue Cancer. Front Oncol 2017;7:191.

10. Zhou XL, Junhua WU, Wang X, et al. Integrated microRNA-mRNA analysis revealing the potential roles of microRNAs in tongue squamous cell cancer. Molecular Medicine Reports 2015;12:885-94.

11. Lin Z, Sun L, Chen W, et al. miR-639 regulates transforming growth factor beta-induced epithelialmesenchymal transition in human tongue cancer cells by targeting FOXC1. Cancer Sci 2014;105:1288-98.

12. Hulin JA, Tommasi S, Elliot D, et al. MiR-193b regulates breast cancer cell migration and vasculogenic mimicry by targeting dimethylarginine dimethylaminohydrolase 1 . Sci Rep 2017;7:13996.

13. Xu C, Liu S, Fu H, et al. MicroRNA-193b regulates proliferation, migration and invasion in human hepatocellular carcinoma cells. European Journal of Cancer 2010;46:2828-36.

14. Guo SL, Zhu ST, Cheng R, et al. Akt pathway inhibited the proliferation of gastric cancer cells by regulating miR-193b. Journal of Clinical \& Experimental Medicine 2016;15:1449-51.

15. Jin X, Sun Y, Yang H, et al. Deregulation of the MiR193b-KRAS Axis Contributes to Impaired Cell Growth in Pancreatic Cancer. Plos One 2015;10:e0125515.

16. Campbell KJ, Tait SWG. Targeting BCL-2 regulated apoptosis in cancer. Open Biol 2018. doi: 10.1098/rsob.180002.
17. Du B, Shim JS. Targeting Epithelial-Mesenchymal Transition (EMT) to Overcome Drug Resistance in Cancer. Molecules 2016;21:965.

18. Yang W, Liu Y, Gao R, et al. HDAC6 inhibition induces glioma stem cells differentiation and enhances cellular radiation sensitivity through the $\mathrm{SHH} / \mathrm{Gli1}$ signaling pathway. Cancer Lett 2018;415:164-76.

19. Nie W, Ge HJ, Yang XQ, et al. LncRNA-UCA1 exerts oncogenic functions in non-small cell lung cancer by targeting miR-193a-3p. Cancer Lett 2016;371:99-106.

20. Guo F, Luo Y, Mu YF, et al. miR-193b directly targets STMN1 and inhibits the malignant phenotype in colorectal cancer. Am J Cancer Res 2016;6:2463-75.

21. Rauhala HE, Jalava SE, Jarkko I, et al. miR-193b is an epigenetically regulated putative tumor suppressor in prostate cancer. Int J Cancer 2010;127:1363-72.

22. Li J, Kong F, Wu K, et al. miR-193b directly targets STMN1 and $\mathrm{UPA}$ genes and suppresses tumor growth and metastasis in pancreatic cancer. Mol Med Rep 2014;10:2613.

23. Nyhan MJ, O'Donovan TR, Boersma AWM, et al. MiR$193 \mathrm{~b}$ promotes autophagy and non-apoptotic cell death in oesophageal cancer cells. Bmc Cancer 2016;16:101.

24. Kaukoniemi KM, Rauhala HE, Scaravilli M, et al. Epigenetically altered miR-193b targets cyclin D1 in prostate cancer. Cancer Med 2015;4:1417-25.

25. Yilmaz M, Christofori G. EMT, the cytoskeleton, and cancer cell invasion. Cancer Metastasis Rev 2009;28:15-33.

26. Li XF, Yan PJ, Shao ZM. Downregulation of miR-193b contributes to enhance urokinase-type plasminogen activator (uPA) expression and tumor progression and invasion in human breast cancer. Oncogene 2009;28:3937-48.

27. Han D, Wang J, Cheng G. LncRNA NEAT1 enhances the radio-resistance of cervical cancer via miR-193b-3p/ CCND1 axis. Oncotarget 2017;9:2395-409.

28. Mao K, Zhang J, He C, et al. Restoration of miR-193b sensitizes Hepatitis B virus-associated hepatocellular carcinoma to sorafenib. Cancer Lett 2014;352:245-52.

29. Long J, Ji Z, Jiang K, et al. miR-193b Modulates Resistance to Doxorubicin in Human Breast Cancer Cells by Downregulating MCL-1. Biomed Research International 2015;2015:1-9.

Cite this article as: Jiang $\mathrm{L}, \mathrm{He} \mathrm{C}$, Zhang $\mathrm{X}$, Chen $\mathrm{Y}, \mathrm{Li}$ G. MiR-193b-5p inhibits proliferation and enhances radiosensitivity by downregulating the AKT/mTOR signaling pathway in tongue cancer. Transl Cancer Res 2020;9(3):18511860. doi: $10.21037 /$ tcr.2020.02.14 